\title{
A New Method of Measuring Retinal Vessel Diameters from Fundus Photographs Based on Retinal Structure and Light Reflection
}

This paper was downloaded from TechRxiv (https://www.techrxiv.org).

\section{LICENSE}

CC BY 4.0

SUBMISSION DATE / POSTED DATE

02-10-2021 / 07-10-2021

\section{CITATION}

Wakebe, Satoshi; Yamasue, Kotaro; Itoh, Hidetoshi (2021): A New Method of Measuring Retinal Vessel Diameters from Fundus Photographs Based on Retinal Structure and Light Reflection. TechRxiv. Preprint. https://doi.org/10.36227/techrxiv.16726555.v1

$\mathrm{DOI}$ 


\section{A new method of measuring retinal vessel diameters from fundus photographs} based on retinal structure and light reflection

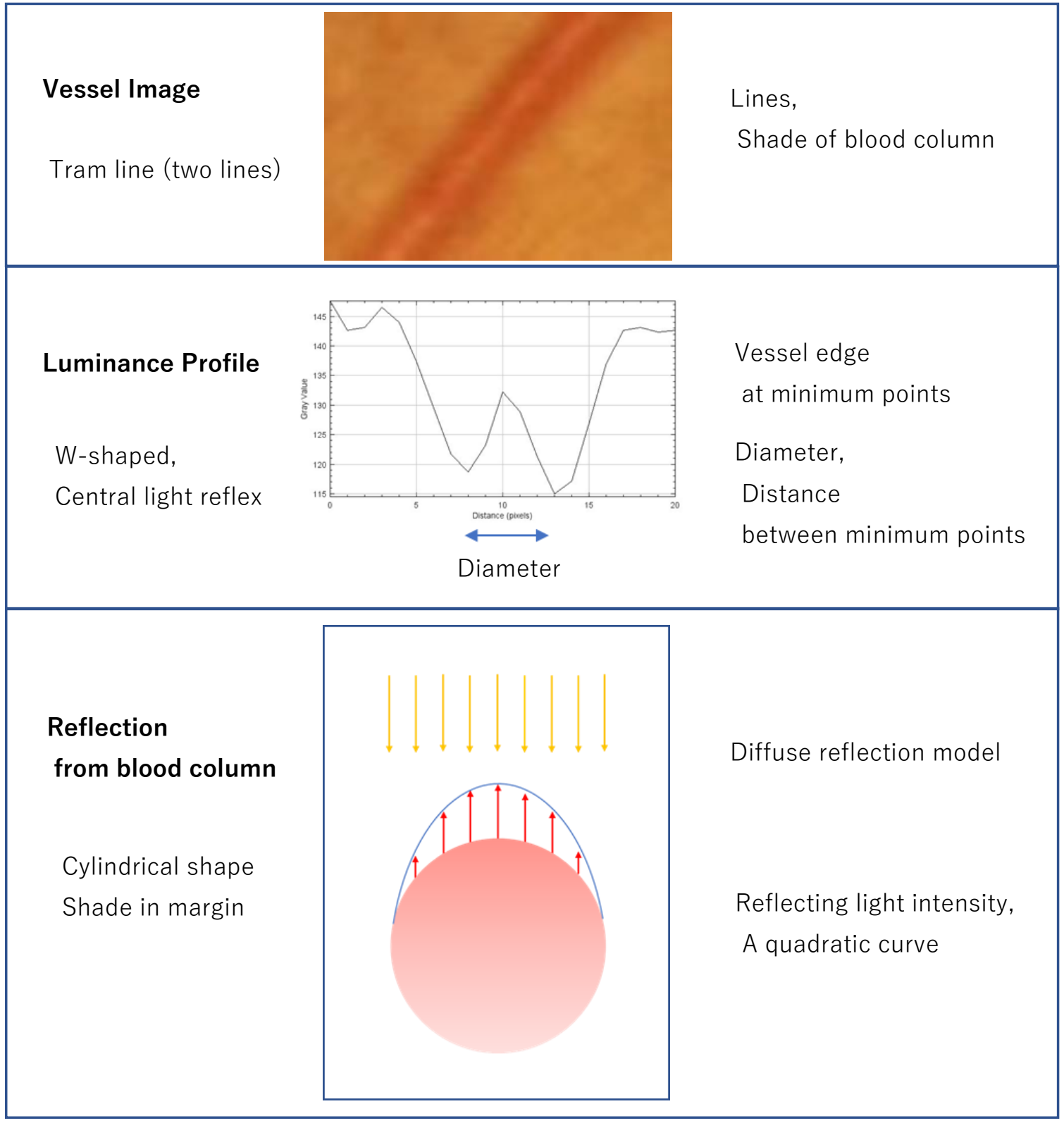

A simple method, small measurement error, easy to automate, no use of mathematical models 


\title{
A New Method of Measuring Retinal Vessel Diameters from Fundus Photographs Based on Retinal Structure and Light Reflection
}

\author{
Satoshi Wakebe*0000-0002-1049-3844, Kotaro Yamasue, and Hidetoshi Itoh
}

\begin{abstract}
Goal: We show validity of a new method and software for measuring retinal vessel diameters. Methods: The diameters are measured as distance of between two minimum points in the luminance profile. The analysis of vascular images and the diffuse reflection model of a cylinder lead the validity of the method. The software was used to evaluate repeated measurement at the same measurement point of the same person. Results: The central reflex of blood vessel in retinal images is the diffuse reflection from the cylindrical blood column, resulting in a W-shaped profile and two minimal points. The simulated image was similar to that of the blood vessel. The coefficient of variation of the diameters of repeated with the software was small $(\leq \mathbf{0 . 1 0})$ and suggests high accuracy of each measurement. Conclusions: The method was reasonable in optics, and reproductive with the software and can be used as a measurement method.
\end{abstract}

Index Terms-Central light reflex, cylindrical shape, fundus images, luminance profile, vessel width.

Significance Statement - The central reflex plays a significant role in the measurement of blood vessel diameter. The proposed method is simple, has small measurement error, and is easy to automate.

\section{INTRODUCTION}

$\mathrm{W}$ E measure the diameter of retinal blood vessels with fundus photographs to investigate the relationship with lifestyle-related diseases such as hypertension. In this paper, we present a new method for measuring the blood vessel diameter and a theoretical basis focusing on the cylindrical shape of the blood vessel, and evaluated the measurements.

Blood vessels are often seen as two lines in fundus photographs. The luminance profile of a vessel transversal line is W-shaped (Fig. 1a). ImageJ [1], a shareware image analysis software, was used for the image analysis. In conventional measurement of blood vessel diameter, the blood vessel edge is defined outside the minimum points in the profile. The inverted $\mathrm{V}$-shaped part in the center is due to the reflection from the blood vessel, but as the central reflex, it was considered to be an obstacle in measurement [2]. Many attempts have been made to determine the point of the

Manuscript submitted June 2, 2021. This work was supported by the Kanagawa Public Health Association under 2015 Grant.

*Satoshi Wakebe is with Oimachi Clinic, 409-5 Kamioi, Oi-machi, Ashigarakami-gun, Kanagawa 2580016 Japan, and the Department of Ophthalmology and Visual Science, Yokohama City University, Yokohama vascular edge. To emphasize contrast in a blood vessel edge, in some cases, image data without the $\mathrm{R}$ component or only the $\mathrm{G}$ component of the RBG image were used.

In the new measurement method presented, we found significance in the central reflex and utilized it. The image with central reflex looks like two lines, and the profile is $\mathrm{W}$ shaped. The inverted V-shaped part in the center of the $\mathrm{W}$ shape is an image of the blood vessel, which is the reflected light from the cylindrical blood column. The dark part of the margin corresponds to the edge of the blood column. So we considered the distance between the edges is the diameter of the blood vessels.

Fig. 1b presents the computer graphics of a cylinder containing columnar stereoscopic information in their shade. It resembles a blood vessel image in a fundus photograph.

Therefore, using the theory of diffuse reflection of optics, we derived a function that expresses the luminosity of the

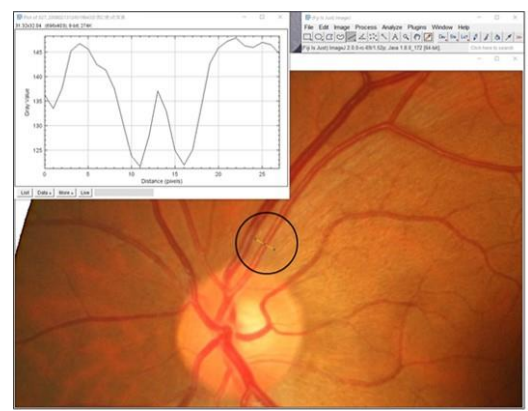

(a)

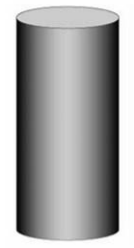

(b)
Fig. 1. Blood vessel image in fundus photograph and a cylinder image in computer graphics. (a) Blood vessels and luminance profile (artery). The blood vessels appear as two lines, and the luminance profile at the transversal line is $\mathrm{W}$-shaped. (b) Representation of a cylinder in computer graphics. Similar to a blood vessel image, the edges look like lines.

observed light reflected by a cylinder. This function formula is a quadratic function. The simulated image closely approximates the blood vessel image, which looks like a tram line (two lines). Thus, we postulated that the characteristics of the blood vessel image in the fundus photograph are derived from the cylindrical shape. The blood vessel wall is transparent, and the blood vessels look like images of blood column, based on the light scattered by red blood cells. The

2360004 Japan (correspondence e-mail: wakebes7@gmail.com). Kotaro Yamasue is with the Unit of Public Health and Preventive Medicine, Yokohama City University School of Medicine, Yokohama 2360004 Japan. Hidetoshi Itoh is with AT System Co., Ltd., 2897 Uchino, Kita-ku, Hamamatsu-shi, Shizuoka 4340044 Japan. 
distance between the minimum points is appropriate for use as the blood column diameter, that is, the diameter of the blood vessel lumen.

The newly developed blood vessel diameter measurement software measures the distance between the minimum points and can semi-automatically measure the series images of the same person at the same point. The 6-year annual measurements at the same point of same person showed little variation. As the minimum point is clear and easy to obtain, small measurement error and it is easy to automate. A mathematical model was not used for this method.

\section{MATERIALS AND METHODS}

We used the digital fundus images of participants captured in medical check-up using a non-mydriatic retinal camera (CR-DG10, Canon Inc., Tokyo, Japan). Written informed consent for participation was obtained from all the participants. The study was carried out in compliance with the Helsinki Declaration and was approved by the Ethics Committee of Yokohama City University under number A150122011.

\section{A. Luminance Profile and Anatomy of Retinal Blood Vessels}

\section{1) Luminance Profile of Blood Vessel Image}

Blood vessel images that have a central reflex and appear as two lines often have a $\mathrm{W}$-shaped luminance profile at the transversal line (Fig. 1a).

The inverted V-shaped part at the center of the W-shape is an image of the blood vessel itself. The brightness is highest at the center and decreases toward the edges. The part containing the minimum point of the $\mathrm{W}$-shape looks like a line. The central reflex, a W-shape with two minimum points, and two lines of the image is three sides of the same thing. The central reflex can be explained by the diffuse reflection phenomenon.

\section{2) Anatomy of Retinal Blood Vessels}

The main trunk of blood vessels lies on the top of the retina, in the nerve fiber layer of the innermost layer of the retina [3]. The retina is transparent except for the retinal pigment epithelium in the outermost layer [4]. The fundus image is a two-dimensional image obtained from the reflected light of the retinal tissue formed by irradiation [3]. The vessel wall is light transparent, and blood columns can be observed [5].

Red blood cells, which scatter light, are the largest component in the blood, having a disk-like shape and a red pigment. Blood columns are observed as scatter light from the red blood cells.

\section{B. Position of Blood Vessel Edge}

1) Conventional Measurement Method and Position of Blood Vessel Edge

Various methods have been devised to determine the position of blood vessel edges. In blood vessels appearing as two lines, the vessel edge was determined outside the minimum point of the $\mathrm{W}$-shape. The following measurement methods were attempted: the half-height method, which obtains the midpoint of the difference in luminance between the blood vessel and the retina; the Gaussian method, which approximates the luminance profile with a Gaussian distribution and uses the inflection point as the edge; the
Sobel operator of the algorithm, which determines the boundary; and the sliding linear regression filter that identifies edge positions by means of linear regression [6] [7] [8] [9]. In each method, the boundary was located on the outside.

Therefore, it was important to strengthen the contrast of the blood vessel to the background [7]. In some cases, the image without the R component of the RBG image [3] [9] or with the $\mathrm{G}$ component only [6] [11] or the $\mathrm{G}$ component weighted was used [10]. Moreover, since the central reflex interferes with fitting the model to the cross-section profile, the double Gaussian method of the devised model was used [6] [8].

2) Luminance Profile of the Blood Vessel and RBG Image Components

Fig. 2 shows the luminance profile of each component of the RGB image of the blood vessel (artery). Each RGB component differs on the outside and inside of the $\mathrm{W}$-shaped minimum points. Here, the inverted V-shaped part corresponds to the blood column.

Because the $\mathrm{R}$ component is high on both the outside and the inside, and the $\mathrm{G}$ component is high on the outside and low on the inside, using an image without the $\mathrm{R}$ component or using only the $\mathrm{G}$ component can possibly make the outside stand out. In the inner inverted V-shaped part, the $\mathrm{G}$ component has a higher peak than the other components, suggesting scattering by red blood cells.

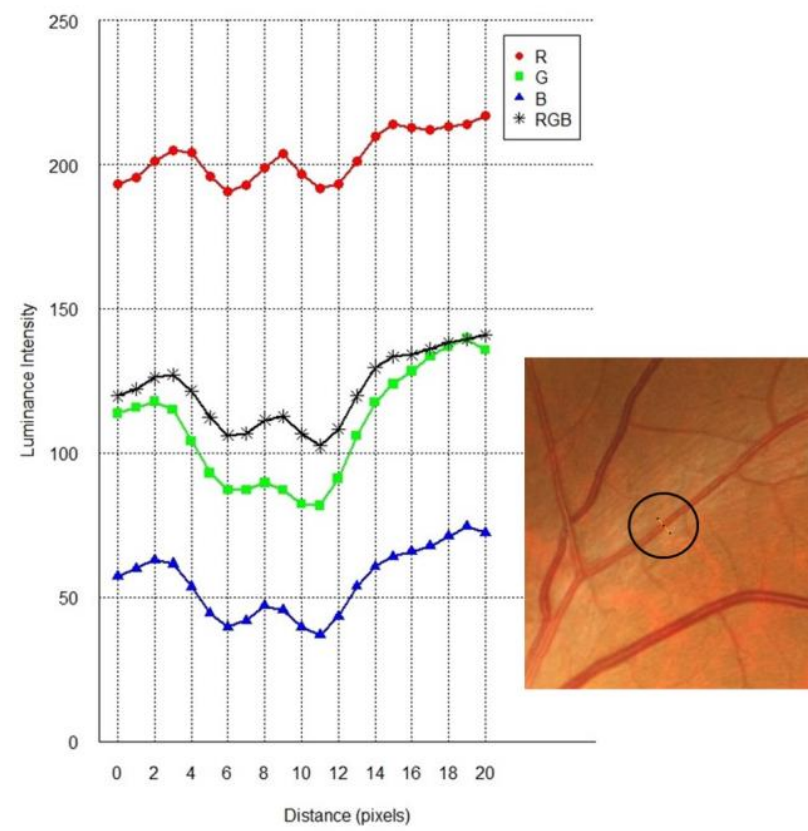

Fig. 2. Luminance profile for each component of the RBG image (artery), and the photograph. Each component of the RGB image differs on the outside and inside of the minimum points of the $\mathrm{W}$-shape. The $\mathrm{R}$ component is high on both the outside and the inside, and the $\mathrm{G}$ component is high on the outside and low on the inside. The high inner peak in the $\mathrm{R}$ component suggests scattering by red blood cells. $R G B=(R+G+B) / 3$.

\section{Computer Graphic Image of a Cylinder}

The computer graphic image of a cylinder is similar to a blood vessel image. The shade on the edge of the cylinder appears to be a line (Fig. 1b). It looks like a line because the 
illusion of the brain emphasizes the difference in shading and makes it appear to have boundaries [12]. In general, an image obtained by shining a directional light on a dull solid follows the rule of diffuse reflection. The image is a two-dimensional plane, but it contains information on the shape of the solid and hence the solid can be imagined. Computer graphic images represent virtual stereoscopic information.

D. Simulation with a Diffuse Reflection Model of a Cylinder

1) Derivation of the Function in the Diffuse Reflection Model of the Cylinder

In the fundus camera, the photographed light hits the blood column, and the reflected light forms an image. In the reflection model, it can be expressed as

reflected light $=$ diffuse reflection + specular reflection [13] .

The reflected light corresponds to the central reflex. On a dull three-dimensional surface, the diffuse reflection component is large, and the specular reflection, i.e., the highlight is small. Here, we derived a function that expresses the reflected light when the irradiation light illuminates the curved surface of the cylinder and diffusely reflects, and an image was then created from the obtained function.

In the diffuse reflection model of a cylinder, the reflected light $I$ is considered in the same direction as the incident light $I_{0}$ (Fig. 3a).

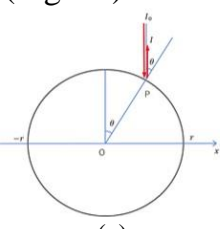

(a)

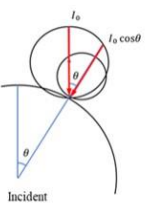

(b)

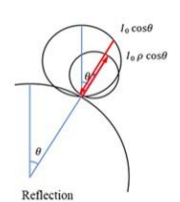

(c)

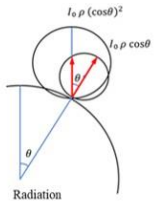

(d)
Fig. 3. Diffuse reflection model of a cylinder. (a) The incident light $I o$ and the reflected light $I$ at point $\mathrm{P}$ on circle $\mathrm{O}$ of the cross section of the cylinder. $I$ and $I_{0}$ are luminous intensity. $\theta$ is the angle of incidence with the normal $(-\pi / 2<\theta<\pi / 2)$, and $r$ is the radius. (b) Incident from angle: $\times \cos \theta$, according to the oblique incident light characteristics (Rambert's cosine law). (c) Attenuation due to reflection: $\times \rho$, diffuse reflection. (d) Radiation from curved surface: $\times \cos \theta$, the diffuse reflection in the oblique direction (Rambert plane). $\theta$ in this case indicates the observation direction but is the same as the incident angle, where $\rho$ is the reflectance of the diffuse reflection.

The relationship between the reflected light $I$ and the incident light $I_{0}$ depends on the coefficients of the incident, reflection and radiation (Fig. 3b,3c,3d). Taking the product of the coefficients, the function is

$$
I=I_{0} \rho(\cos \theta)^{2} \text {. }
$$

Information on the shape of the cylinder is included in this function as the radiation of the luminous flux.

2) Simulation by the Diffuse Reflection Model of the Cylinder

The function is converted from polar to $x y$ coordinates in order to draw a simulation image.

Here,

$y=I, x=r \sin \theta$, and $(\sin \theta)^{2}+(\cos \theta)^{2}=1$.

From (1), the following relationship between $x$ and $y$ is obtained:

$$
y=I_{0} \rho\left(-\frac{1}{r^{2}} x^{2}+1\right) .
$$

The graph showing the relationship between intensity $y$ of the reflected light and position $x$ is a quadratic curve (parabola). It is an upward convex that passes through the vertices $\left(0, l_{0} \rho\right)$ and two points on the $x$-axis, $(r, 0)$ and $(-r$, $0)$.

Using this function, we simulated an illuminated image of a cylinder. The luminance profile is $\mathrm{W}$-shaped, and the image resembles the blood vessel image of the fundus photograph, which looks like two lines (Fig. 4).
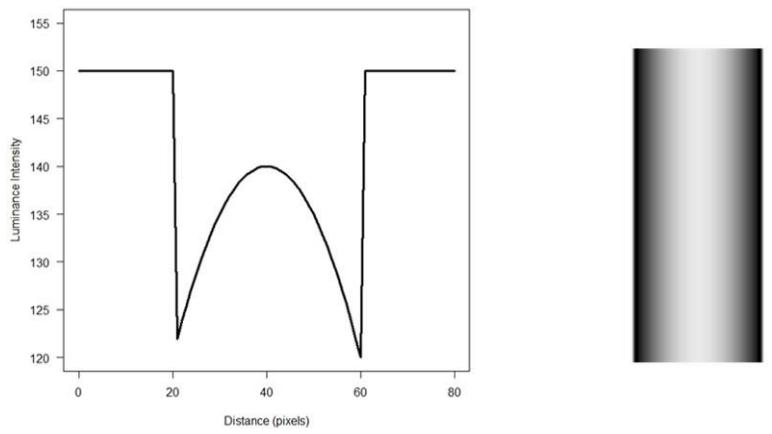

Fig. 4. Diffuse reflection model of the cylinder: the graph and the image. It is similar to the luminance profile graph and the blood vessel image of the retinal blood vessels in the fundus photograph.

\section{E. Introducing the Blood Vessel Diameter Measurement Method}

In this method, the blood vessel diameter is measured by approximating a parallelogram. The diameter is measured from the area of a parallelogram that approximates the corresponding portion of the vessel within a rectangular region of interest (ROI) (Fig. 5).

The vessel diameter $d$ is calculated by

$$
d=S / \ell \text {, }
$$

where the area $S$ is surrounded by $\mathrm{ABDC}$ in the ROI and is calculated by integrating the following: a $i$, which is the distance between the minimum points in the $x$-axis direction of the $i$-th row $i$, which is the number of rows, and $l$, which is the length of the long axis of the blood vessel in the ROI. $\mathrm{AC}$ and $\mathrm{BD}$ are the lines connecting the positions of the minimum values. When the blood vessel runs across the ROI from side to side, it is calculated in the $y$-axis direction. 

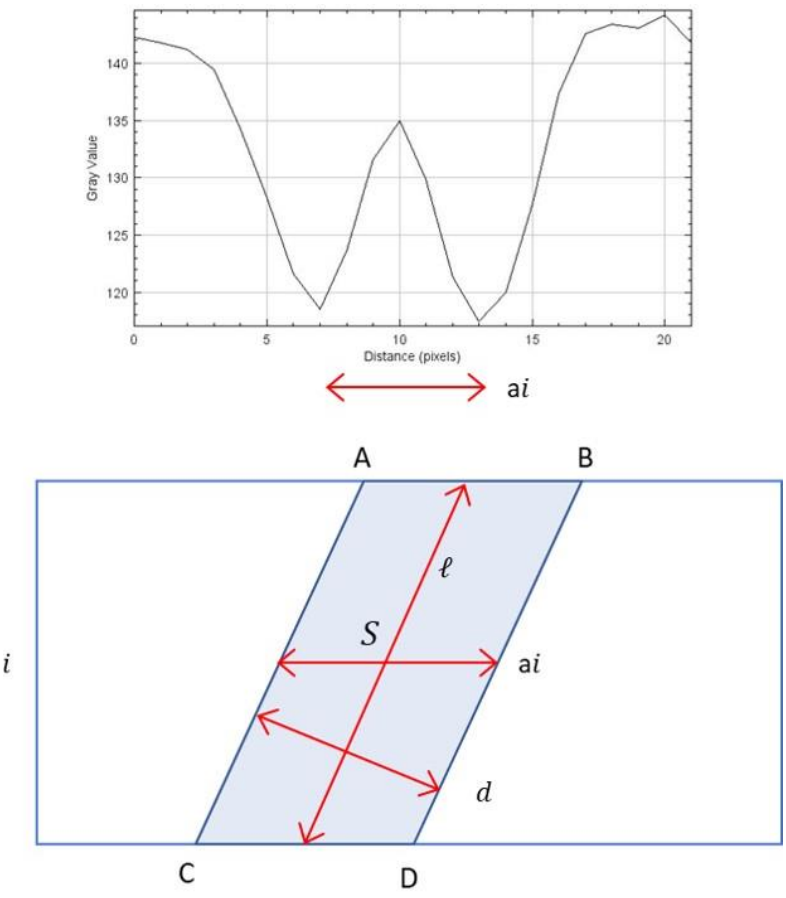

Fig. 5. Calculation of the blood vessel diameter by approximation of a parallelogram.

\section{RESULTS}

\section{A. Measurement with Semi-automatic Measurement} Software

The measurements were performed using a newly developed semi-automatic measurement software, CalcSize. The software measures the blood vessel diameter at the same position using a plurality of fundus photographs of the same person taken repeatedly.

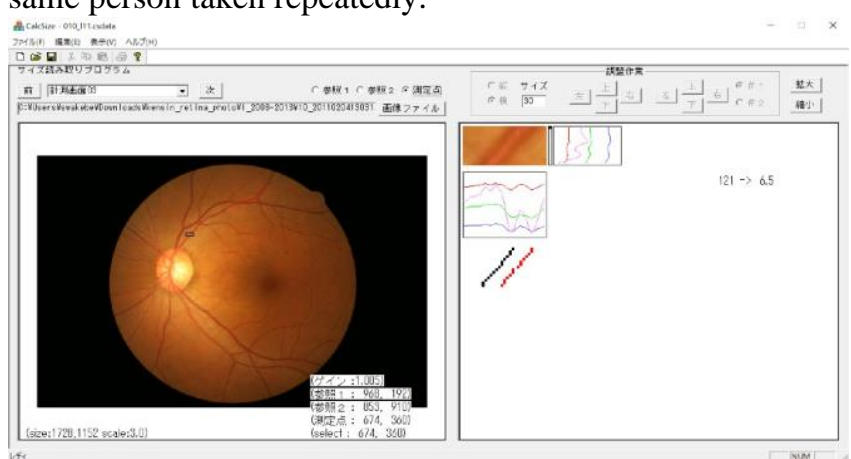

Fig. 6. Screen of the developed measurement software, CalcSize. Allocate the measurement area with a rectangle in the fundus image on the left side of the screen. The right side of the screen shows the 15 minimum points (black and red points) detected in the measurement area, and the blood vessel diameter is calculated.

Within the ROI, the distance between the minimum points in the $x$-axis direction was measured for 15 points at 1 -pixel intervals along the length of the blood vessel, and the area of the parallelogram was integrated. The blood vessel diameter corresponds to the height obtained by dividing the area of the parallelogram by the base of the inclined blood vessel length (Fig. 6).

In addition, the diameter at the same position in a series of photographs of the same person can be automatically measured by collating two points of blood vessel bifurcations as reference points in each image. The morphology of retinal vessels does not change in one's lifetime similar to fingerprints.

For the brightness value of the RGB image, the formula $R G B$ (gray value) $=(R+G+B) / 3$ was used.

\section{B. Software Measurement Results}

The blood vessel diameter was measured using the 6-year fundus photographs of the left and right eyes of five people that were taken clearly. The mean of diameter and the standard error of the predicted value in the regression and the coefficient of variation were calculated for six series of photographs taken once annually over 6 years. The standard error of the predicted value was calculated using the STEYX function in Microsoft Excel. The coefficient of variation is defined as the ratio of the standard error to the mean. Using the same photographs, the arteries and veins were measured at multiple locations, with a total of 44 points.

The characteristics of the mean diameter and the coefficient of variation over 6 years are shown in Table I. The slope of the regression lines indicates the amount of change in the blood vessel diameter per year, and the standard error of the predicted value in the regression indicates the variation over 6 years. In addition, the coefficient of variation can be used to compare the measured values of the blood vessels of different diameters.

TABLE I

CHARACTERISTICS OF MEAN DIAMETER AND COEFFICIENT OF VARIATION OVER 6 YEARS

\begin{tabular}{|l|l|r|r|r|r|}
\hline \multicolumn{2}{|c|}{} & $\begin{array}{c}\text { Mean Diameter } \\
\text { over 6years }\end{array}$ & $\begin{array}{c}\text { Standard Error } \\
\text { of Predicted } \\
\text { Value }\end{array}$ & $\begin{array}{c}\text { Slope of } \\
\text { Regression } \\
\text { Lines }\end{array}$ & $\begin{array}{c}\text { Coefficient } \\
\text { of Variation }\end{array}$ \\
\hline \multirow{3}{*}{$\begin{array}{c}\text { Artery } \\
(\mathrm{n}=28)\end{array}$} & Minimum & 4.7 & 0.10 & -0.09 & 0.014 \\
\cline { 2 - 6 } & Maximum & 8.3 & 0.81 & 0.16 & 0.116 \\
\cline { 2 - 6 } & Average & 6.3 & 0.29 & 0.01 & 0.047 \\
\cline { 2 - 6 } & Median & 6.3 & 0.27 & 0.00 & 0.041 \\
\hline \multirow{3}{*}{$\begin{array}{c}\text { Vein } \\
(\mathrm{n}=16)\end{array}$} & Minimum & 5.4 & 0.13 & -0.12 & 0.018 \\
\cline { 2 - 6 } & Maximum & 8.4 & 0.72 & 0.11 & 0.109 \\
\cline { 2 - 6 } & Average & 7.0 & 0.33 & 0.01 & 0.048 \\
\cline { 2 - 6 } & Median & 6.8 & 0.28 & 0.00 & 0.044 \\
\hline
\end{tabular}

The unit of blood vessel diameter is shown in pixels.

The slope of the regression line of the repeated data over 6 years is in the range of approximately -0.1 to +0.1 (pixel/year) for both arteries and veins, and their average value is as low as 0.01 . Therefore, the change over 6 years was small, and it could be used as data for repeated measurements. The average coefficient of variation ( \pm standard error) was $0.047 \pm 0.024$ for arteries and $0.048 \pm$ 0.024 for veins. The graph of the relationship between the individual coefficients of variation and blood vessel diameter is shown in Fig. 7. The magnitude of the coefficient of variation was low at $\leq 0.10(10 \%)$, and there was little variation. In addition, the coefficient of variation was not affected by blood vessel diameter and arteriovenous distinction. 


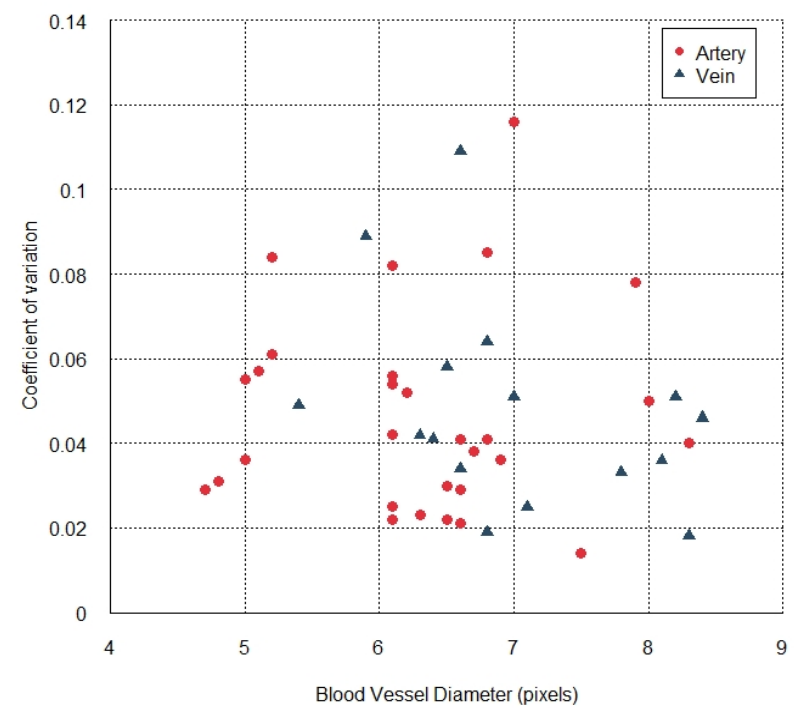

Fig. 7. Relationship between the coefficient of variation (CV) and the blood vessel diameter. The plots show $\mathrm{CV}$ in the range below 0.1 , regardless of different blood vessel diameters.

\section{DISCUSSION}

\section{A. Evaluation of Coefficient of Variation and Software} Accuracy

The reason for the coefficient of variation to be as small as $\leq 10 \%$ may be attributed to the accumulation of the following series of results: accuracy at measurement points, accuracy of repeated measurements using the software, and little change in participants due to aging and imaging conditions.

1) Accuracy and Measurement Error at Individual Measurement Points.

This study did not compare with the measurement of other methods, as comparison with similar methods is not important. However, the accuracy of this measurement method can be explained as follows: at the measurement point the average value was calculated from 15 measured values. The minimum point was selected from the brightness value, and the number of pixels was counted to obtain the measured value. The minimum point was clear from the brightness profile. Because raw numerical values were used, there was no error due to approximation, and the propagation of error due to calculation is small.

By measuring the average value of 15 points, the measurement accuracy was improved by one order of magnitude. The distance between the two minimum points was less than 10 pixels, which is one significant digit. The measurement of 15 point has increased the number of significant figures to two digits.

\section{2) Accuracy of Repeated Measurement by the Software}

The value of the coefficient of variation in the data of repeated measurements for the same point of the same person was small. From this result, we were convinced that the reproducibility of repeated measurements was sufficient, despite a shooting interval of 1 year. The measurement equipment and shooting environment did not change. Even in different diameters or arteriovenous distinction, the repeated measurement result was mostly same. Furthermore, the coefficient of variation that is the accumulation of the above series of results was as low as $\leq 10 \%$. Therefore, the accuracy of both measurement at each point and repeated measurements was demonstrated, with this software.

3) Small Changes of Over Time in the Participants and Shooting Conditions

We used 6-year repetitive data annually. The slope of the regression line was small, and the changes over time and changes in the shooting conditions were small.

\section{B. Limitations}

In this paper, the absolute value of blood vessel diameter was not calculated, but the number of pixels in the image was measured. In the repeated measurement with the software, there was no problem in comparison in the same person. The study did not measure the true value. It is expected that the measured value will be slightly lower than the conventionally measured value. We have not verified the extent to which the measured values of the same position make a difference based on the measuring equipment and shooting conditions. In addition, it is not clear whether the ratio of the measured value to the true value differs depending on the blood vessel diameter and arteriovenous distinction. Furthermore, the measured value with another device is not always the true value, even in optical coherence tomography. If the blood vessel image does not look like two lines and the luminance profile is U-shaped instead of W-shaped, measurement is not possible with this method. This study only measured the image data of healthy adults, and analysis of fundus photographs with arteriosclerotic and hypertensive changes is expected using this method.

\section{Future Prospects}

The blood vessel image of the retina contains threedimensional information. In the analysis of retinal images, an understanding of retinal structure and optics may yield further information. Adding the effect of ambient light from the neural retina around the blood vessel to the diffuse reflection of the irradiation light, the simulation of blood vessel images may be close to the actual luminance profile of the blood vessel.

\section{CONCLUSION}

We proposed that the minimum point in the profile is the blood vessel edge and the distance between them is the blood vessel diameter. The central reflex, which had not been utilized, represents cylindrical three-dimensional information in the blood vessel image. This assumption is theoretically valid by the brightness analysis of the fundus image and simulation of diffuse reflection model of the cylinder. The developed software works with an extremely simple measurement procedure, has small measurement error, and is easy to automate.

\section{ACKNOWLEDGMENT}

We thank the staff of the Kanagawa Health Service Association for their medical check-up data. We are grateful to Professor Tomoki Hamagami of the Graduate School of Engineering, Yokohama National University, and Mr. Kosuke Ito, a graduate student (at that time), for guiding us on the basics of image analysis. Dr. Takehito Ishido, Fujisawa 
City Hospital Ophthalmology, answered many of my questions as an ophthalmologist. We would like to thank Editage (www.editage.com) for English language editing.

\section{REFERENCES}

[1] W. S. Rasband. (1997-2018). ImageJ. [Online]. Bethesda, MD: U.S. National Institutes of Health. Available: ttps://imagej.nih.gov/ij/

[2] H. Li, W. Hsu, M.L. Lee, T. Y. Wong, "Automatic grading of retinal vessel caliber," IEEE Trans. Biomed. Eng., vol. 52, no. 7, pp. 1352-1355, 2005.

[3] M. D. Abràmoff, M. K. Garvin, M. Sonka, "Retinal imaging and image analysis," IEEE Rev. Biomed. Eng., vol. 3, pp. 169-208, 2010.

[4] M. Batterbury, C. Murphy, "Anatomy and physiology: inner eye," in Ophthalmology, An Illustrated Colour Text, 4th ed. Edinburgh, UK Elsevier, ch. 2, pp. 4-5, 2018.

[5] H. K. Walker, W. D. Hall, J. W. Hurst, "The funduscopic examination," in Clinical Methods: The History, Physical, and Laboratory Examinations (H. Schneiderman, editor), 3rd ed. Boston, MA: Butterworths, ch. 117, pp. 573-580, 1990

[6] J. Lowell, A. Hunter, D. Steel, A. Basu, R. Ryder, R. L. Kennedy, "Measurement of retinal vessel widths from fundus images based on 2-D modeling," IEEE Trans. Med. Imag., vol. 23, no. 10, pp. 1196-1204, 2004.

[7] E. Trucco, L. Ballerini, D. Relan, A. Giachetti, T. MacGillivray, K. Zutis, C. Lupascu. Novel VAMPIRE algorithms for quantitative analysis of the retinal vasculature. Presented at IEEE ISSNIP 2013: Biosignals and Biorobotics Conference, 1-4: Biosignals and Robotics for Better and Safer Living, 2013.

[8] M. M. Fraz et al., "QUARTZ: quantitative analysis of retinal vessel topology and size - an automated system for quantification of retinal vessels morphology," Expert Syst. Appl., vol. 42, no. 20, pp. 7221-7234, 2015.

[9] N. Chapman et al., "Computer algorithms for the automated measurement of retinal arteriolar diameters," Br. J. Ophthalmol., vol 85, no. 1, pp. 74 79, 2001.

[10] M. D. Knudtson et al., "Variation associated with measurement of retinal vessel diameters at different points in the pulse cycle," $B r . J$. Ophthalmol., vol. 88, no. 1, pp. 57-61, 2004.

[11] A. Bhuiyan et al., "Retinal artery and venular caliber grading: a semiautomated evaluation tool," Computers in Biology and Medicine, vol. 44 pp. $1-9,2014$

[12] A. Fiorentini, "Mach band phenomena," in Visual Psychophysics (D. Jameson, L.M. Hurvich, editor). Springer-Verlag: Berlin, Heidelberg, Germany, ch. 8, pp. 188-201, 1972.

[13] A. Ryer. (1997). Light Measurement Handbook. [Online]. Newburyport, MA: International Light Inc. Available: https://www.intllighttech.com/light-measurement-handbook 